\section{Association between persistent tachycardia and tachypnea and in- hospital mortality among non- hypotensive emergency department patients admitted to the hospital}

\author{
Michael A Puskarich', Utsav Nandi', Ben G Long'ㄹ, Alan E Jones ${ }^{1}$ \\ 'Department of Emergency Medicine, University of Mississippi Medical Center, Jackson, MS, USA \\ ${ }^{2}$ School of Medicine, University of Mississippi Medical Center, Jackson, MS, USA
}

Objective Vital sign trends are used in clinical practice to assess treatment response and aid in disposition, yet quantitative data to support this practice are lacking. This study aimed to determine the prognostic value of vital sign normalization.

Methods Secondary analysis of a prospective cohort of adult emergency department (ED) patients admitted a single urban tertiary care hospital. A random sample of 182 days was chosen, and a manual review of all admissions was undertaken. Persistent tachycardia or tachypnea was defined as failure to decrease to a normal value in the ED. Elevated upon admission was defined as an abnormal value at the last set of vital signs documented. The primary outcome was in-hospital mortality.

Results 4,878 patients were enrolled and $4.5( \pm 3.8)$ sets of vital signs were checked per patient. 1,770 patients were tachycardic and 1,499 were tachypneic. Among tachycardic patients, 941 (53\%) were persistently tachycardic and 1,074 (61\%) were tachycardic upon admission. Among tachypneic patients $639(42 \%)$ were persistently tachypneic and 768 (51\%) were tachypneic upon admission. Mortality was higher in patients persistently tachycardic (5.7\% vs. $3.1 \%$, $\mathrm{P}=0.008)$ or tachycardic upon admission (5.5\% vs. $3.0 \%, \mathrm{P}=0.014)$. Similar results were found in tachypneic patients ( $8.3 \%$ vs. $4.5 \%, \mathrm{P}=0.003 ; 7.8 \%$ vs. $4.4 \%, \mathrm{P}=0.006$ ).

Conclusion Persistent tachycardia and tachypnea are associated with an increased risk of mortality in ED patients admitted to the hospital. Further study is necessary to determine if improved recognition or earlier interventions can affect outcomes.

Keywords Vital signs; Tachypnea; Tachycardia; Mortality; Triage

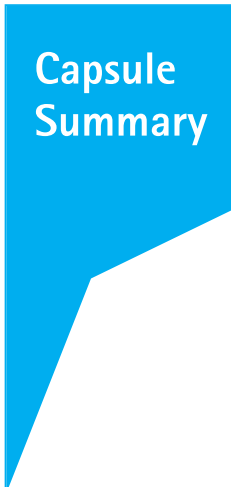

elSSN: 2383-4625

Received: 4 October 2016

Revised: 5 December 2016

Accepted: 16 December 2016

Correspondence to: Alan E Jones Department of Emergency Medicine, University of Mississippi Medical Center, 2500 N State Street, Jackson, MS 39216, USA

E-mail: aejones@umc.edu

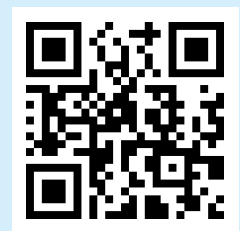

How to cite this article:

Puskarich MA, Nandi U, Long BG, Jones AE. Association between persistent tachycardia and tachypnea and in-hospital mortality among non-hypotensive emergency department patients admitted to the hospital. Clin Exp Emerg Med 2017;4(1):2-9

This is an Open Access article distributed under the terms of the Creative Commons Attribution Non-Commercial License (http:// creativecommons.org/licenses/by-nc/4.0/). 


\section{INTRODUCTION}

There are approximately 136 million emergency department (ED) visits annually of which 17.2 million result in hospital admission. ${ }^{1}$ Such volume creates a need for easy and rapid assessment and triage due to limited resources. Adverse patient outcomes, reduced quality, and impaired access can all be attributed to ED crowding. ${ }^{2}$ Effects downstream of admission, such as intensive care unit crowding, is also a function of capacity challenges that affect patient care and outcomes. ${ }^{3}$ Accurate risk stratification allows for appropriate resource allocation of admitted patients to the necessary level of care for their in-hospital stay. Vital signs are a simple tool that are easily measured at the bedside and provide clinicians with a means for risk stratification during every ED encounter. However, quantitative analysis of these commonly measured clinical variables remain surprisingly understudied. Previous work done by our group has demonstrated that even a single, isolated episode of hypotension is association with increased patient mortality, ${ }_{1}^{4}$ even in the pre-hospital setting ${ }_{1}^{5}$ and the more abnormal the blood pressure in depth and duration, the higher the risk of mortality. ${ }^{6}$

The prognostic and diagnostic value of other vital signs, most notably heart rate and respiratory rate, have been studied most extensively in relationship to the systemic inflammatory response syndrome. Their use for diagnosis and prognosis in this regard are fairly limited in terms of clinical decision making, as they suffer from relatively poor test characteristics. ${ }^{7-9}$ In clinical practice, single measurements of vital signs are rarely used in isolation, particularly among patients with a higher acuity of illness. It is more common in high acuity scenarios for clinicians to evaluate temporal trends of vital signs to aid in determining the response to treatment to help guide disposition decisions, or to assist in determination of the appropriate level of inpatient care. Yet there are little or no objective data to support this practice. Thus a better understanding of the utility of vital sign normalization versus persistence of abnormal values could provide additional, freely available, and clinically useful information to clinicians and researchers. The primary hypothesis of this study was that persistently abnormal heart and respiratory rate among a cohort of heterogeneous ED patients admitted to the hospital would be associated with an increased risk of mortality.

\section{METHODS}

This study was a secondary analysis of a previously published prospective cohort of ED patients presenting to a single, urban, tertiary care ED from July 2004 to June 2005 with > 100,000 yearly visits. The purpose of this parent study was to determine the prognostic value of increasingly frequent episodes of hypotension, and its results have been published previously. ${ }^{6}$ Inclusion criteria of the parent trial were broad and included all patients age $\geq 18$ undergoing hospital admission. Exclusion criteria were trauma to the torso within 24 hours and direct admission bypassing the ED. A random sample of 182 days was chosen, and a manual review of all admissions to the hospital on those days was undertaken to ensure completeness. Patient demographics, comorbidities, and volume of intravenous fluid administration in the ED were recorded. Vital signs were measured and recorded per ED protocol, and all vital signs (up to 15 measurements) were recorded with demographic data into a de-identified database. Upon hospital discharge, in-hospital mortality was recorded.

To be included in the present analysis, subjects were those that had measured tachycardia or tachypnea at ED presentation as well as measurement of at least 2 sets of vital signs. Tachycardia was defined as $\geq 100$ beats/min, tachypnea as $>20$ breaths/min at presentation, and persistent as failure to decrease to a normal ( $<100 \mathrm{bpm}$ or $\leq 20$ breaths/min) level. The primary outcome was in-hospital mortality.

Two separate analyses were performed for each of the two vital signs. In the first analysis, patients were categorized based on the last value of either heart or respiratory rate prior to hospital admission. If those values were normal, the patient was categorized as normal upon admission. If that value was elevated, the patient was categorized as elevated upon admission. To increase the robustness of the analysis, a second analysis was also conducted. In this analysis, patients were categorized into persistently abnormal (every heart rate or respiratory rate measured in the ED was abnormal) versus those that normalized the vital sign (at least one normal heart rate or respiratory rate). In addition, we conducted a post-hoc sensitivity analysis using different cutoffs for the definition of tachycardia and tachypnea using the values of 90 and 110 beats/min, and 18 and 22 breaths/min, respectively. All data analyses were conducted in the entire cohort as well a predefined analysis of only those patients without any episode of hypotension (defined as any measurement of systolic blood pressure $<100 \mathrm{mmHg}$ ) in the ED. A post-hoc analysis was then conducted to examine the potential independent prognostic value of persistent tachycardia and tachypnea in relationship to each other in a subgroup of patients who presented with both tachycardia and tachypnea.

Simple descriptive statistics were utilized to describe the groups, including chi-square, Student's t-test, and Wilcoxson rank sum, as appropriate. Chi-square tests were used to compare mortality rates between groups. In order to assess whether or not 
vital sign normalization was an independent risk factor, a logistic regression model was constructed using in-hospital mortality as the dependent variable. Candidate variables were considered for the model if they differed between survivors and non-survivors $(P<0.10)$, and the model was refined using reverse stepwise elimination. Any episode of hypotension was forced into the final model regardless of statistical significance in order to assess whether vital sign normalization was simply another marker of hemodynamic instability that could be measured more simply through the use of blood pressure. Finally, we developed a posthoc model incorporating Glasgow Coma Score (GCS), which is also readily available clinically. In the post-hoc analysis, a Mantel-Haenszel chi-square was utilized to account for stratification. Commercially available statistical software (StatsDirect ver. 2.7.7, StatsDirect, Cheshire, England; STATA ver. 10.0, StataCorp., College Station, TX, USA) were utilized for data analysis. All tests were two-sided with P-values of $<0.05$ considered significant.

\section{RESULTS}

A total of 4,878 patients were enrolled; 4,164 of whom had at least 2 heart rate measurements, while 3,927 had at least 2 respiratory rates recorded. An average of $4.5( \pm 3.8)$ sets of vital signs were checked per patient.1,770 and 1,499 of these patients were tachycardic and tachypneic at presentation respectively, and formed the cohort of patients eligible for our study. Among these, 1,387 (78\%) and 1,181 (79\%) never had a systolic blood pressure $<100$ and formed our sensitivity analysis cohort of patients without hypotension. The percentage of patients who had persistently abnormal versus normalized vital signs, and the percentage of patients with vital signs that were abnormal versus normal upon admission are summarized Table 1. Patient demographics and clinical characteristics of those patients who had persistently abnormal heart and respiratory rates through their ED stay compared to normalized vitals are summarized in Table 2. Non-Hispanic ethnicity and a history of asthma or chronic obstructive pulmonary disease were associated with persistence of both tachycardia and tachypnea. End stage renal disease and lower white blood cell counts were associated with higher rates of heart rate normalization, while older age and higher intravenous fluid volume in the ED were associated with higher rates of respiratory rate normalization.

In the unadjusted univariate analysis, mortality was significantly higher in patients persistently tachycardic throughout their ED stay (5.7\% \{95\% confidence interval [Cl], 4.2\% to $7.2 \%\}$ vs. $3.1 \%[95 \% \mathrm{Cl}, 1.9 \%$ to $4.3 \%], \mathrm{P}=0.008$ ) and among those that were persistently tachycardic versus those that normalized upon admission (5.5\% [95\% $\mathrm{Cl}, 4.1 \%$ to $6.9 \%]$ vs. $3.0 \%[95 \% \mathrm{Cl}$ $1.7 \%$ to $4.3 \%], \mathrm{P}=0.014)$. Similarly, mortality was higher among persistently tachypneic versus those that normalized their respiratory rate $\left(8.3 \%[95 \% \mathrm{Cl}, 6.1 \%\right.$ to $10.4 \%]$ vs. $4.5 \%\left[95 \% \mathrm{Cl}_{\text {, }}\right.$ $3.1 \%$ to $5.9 \%], P=0.003$ ), and higher among those tachypneic upon admission versus those with a normal respiratory rate $(7.8 \%$ [95\% Cl, $5.9 \%$ to $9.7 \%$ ] vs. $4.4 \%$ [95\% $\mathrm{Cl}, 2.9 \%$ to $5.9 \%$, $\mathrm{P}=0.006)$. Of note, the absolute mortality rates were higher among patients with persistent tachypnea as opposed to tachycardia, and these data are illustrated in Fig. 1. All of these results remained significant after excluding patients with any episode of hypotension ( $P=0.001-0.037)$, though the absolute mortality rates in this group of patients dropped to $1.3 \%$ and $5.5 \%$. Our sensitivity analysis demonstrated similar results for predicting inhospital mortality whether tachycardia was defined as 90 or 110 beats per minute. Similar results were also found when tachypnea was defined as 22 , but not 18 , breaths per minute (data not shown).

Differences between survivors and non-survivors can be found in Table 3, and those variables that differed (as estimated by $\mathrm{P}<0.10$ ) were added as candidate variables to each of the logistic regression models (one for tachycardic and one for tachypneic patients), using in-hospital mortality as the dependent variable. Following model refinement, the final model for tachycardic patients included age, hypertension, end-stage renal disease, white blood count, hemoglobin, bicarbonate, hypotension, and either temporary normalization or normal heart rate upon admission. The final model for tachypneic patients included age, hypertension, white blood count, hemoglobin, bicarbonate, hypotension, and either temporary normalization or normal respiratory rate upon admission. Hypotension remained a significant independent predictor and did not need to be forced into the final model. Other commonly available vital signs, such as temperature and pulse oximetry, were not significant predictors of outcome in the multivariate model, even when categorizing temperature as a binary

Table 1. Number of patients meeting study definitions

\begin{tabular}{lccc}
\hline & Persistently abnormal & Temporarily normalized & Abnormal upon admission \\
\hline Tachycardic $(n=1,770)$ & $941(53)$ & $829(47)$ & $1,074(61)$ \\
Tachypneic $(n=1,499)$ & $639(43)$ & $860(57)$ & $768(51)$ \\
\hline
\end{tabular}

Values are presented as number (\%). 
Michael A Puskarich, et al.

Table 2. Patient demographics and clinical characteristics

\begin{tabular}{|c|c|c|c|c|c|c|}
\hline & \multicolumn{3}{|c|}{ Tachycardia } & \multicolumn{3}{|c|}{ Tachypnea } \\
\hline Sex & & & 0.91 & & & 0.54 \\
\hline Male & 411 (49.6) & $470(50)$ & & 427 (49.8) & $329(51.5)$ & \\
\hline Female & $418(50.4)$ & $471(50.1)$ & & $431(50.2)$ & $310(48.5)$ & \\
\hline African American & $415(50.1)$ & $500(53.1)$ & 0.20 & $439(51.2)$ & $321(50.2)$ & 0.72 \\
\hline Asian & $10(1.2)$ & $5(0.5)$ & 0.12 & $5(0.6)$ & $6(0.9)$ & 0.42 \\
\hline Other & $7(0.8)$ & $13(1.4)$ & 0.29 & $9(0.9)$ & $6(0.9)$ & 0.83 \\
\hline Ethnicity & & & 0.02 & & & 0.02 \\
\hline Hispanic & $42(5.1)$ & $26(2.8)$ & & 32 (3.7) & $10(1.6)$ & \\
\hline End-stage renal disease & $84(8.9)$ & $37(4.5)$ & $<0.01$ & $42(4.9)$ & $44(6.9)$ & 0.10 \\
\hline Cirrhosis/hepatitis & $34(4.1)$ & $56(6)$ & 0.08 & $39(4.5)$ & $31(4.9)$ & 0.77 \\
\hline Stroke & $60(7.2)$ & $55(5.8)$ & 0.24 & $64(7.5)$ & $59(9.2)$ & 0.21 \\
\hline Asthma/COPD & $125(15.1)$ & $191(20.3)$ & $<0.01$ & $168(19.6)$ & $179(28.0)$ & $<0.01$ \\
\hline Malignancy (active) & $42(5.1)$ & $62(6.6)$ & 0.17 & $40(4.7)$ & $35(5.5)$ & 0.67 \\
\hline HIV/AIDS & $61(7.4)$ & $57(6.1)$ & 0.27 & $51(5.9)$ & $29(4.5)$ & 0.24 \\
\hline White blood count, cells per $\mathrm{mm}^{3}$ (SD) & $10.3(5.2)$ & $11.8(8.1)$ & $<0.01$ & $10.4(5.3)$ & $10.9(7.8)$ & 0.12 \\
\hline Hemoglobin, mg/dL (SD) & $12.9(2.5)$ & $12.8(2.4)$ & 0.20 & $12.8(2.3)$ & $12.8(2.4)$ & 0.76 \\
\hline Bicarbonate, mg/dL (IQR) & $25(22-27)$ & $25(22-27)$ & 0.32 & $25(23-28)$ & $25(23-28)$ & 0.95 \\
\hline Creatinine, mg/dL (IQR) & $1.6(0.8-1.5)$ & $1.8(0.8-1.6)$ & 0.50 & $1.1(0.8-1.7)$ & $1.1(0.9-1.6)$ & 0.60 \\
\hline
\end{tabular}

Values are presented as number (\%) unless otherwise indicated.

SD, standard deviation; CHF, congestive heart failure; COPD, chronic obstructive pulmonary disease; HIV/AIDS, human immunodeficiency virus/acquired immunodeficiency syndrome; IQR, interquartile range.
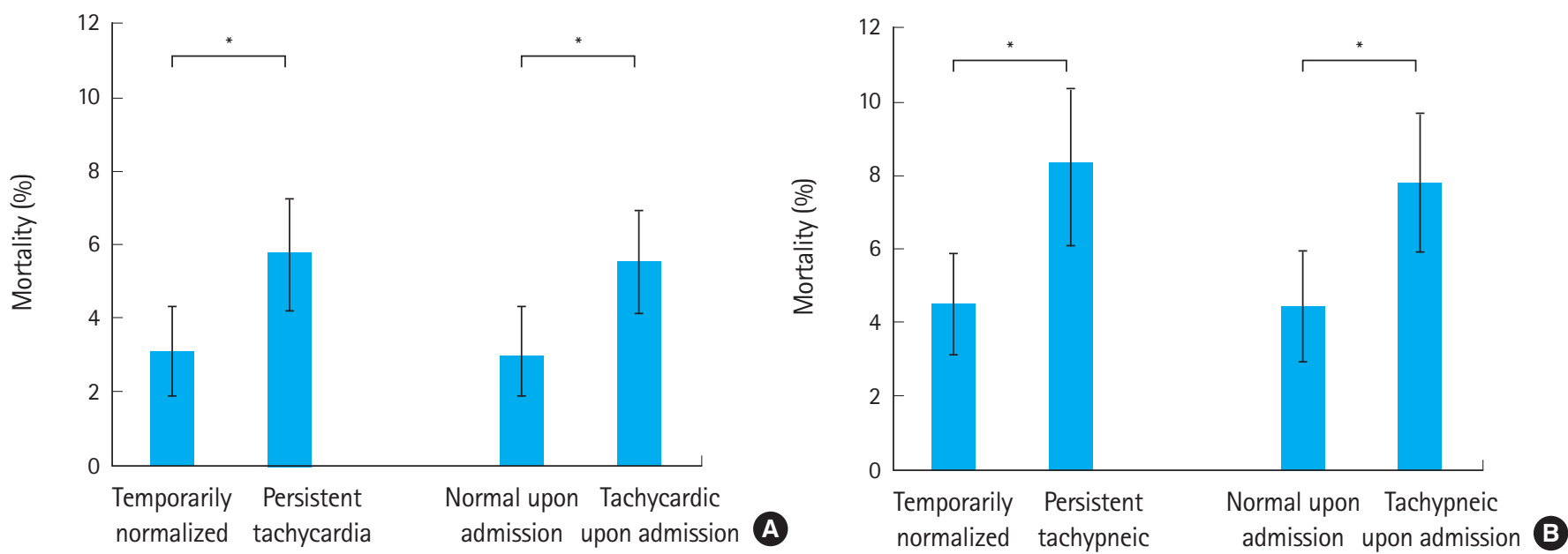

Fig. 1. Relative mortality associated with the persistence of (A) tachycardia and (B) tachypnea. Patients were categorized as to whether they temporarily normalized or were persistently elevated in the emergency department and whether they were persistently abnormal or normalized upon hospital admission. Data are shown with $95 \%$ confidence intervals. ${ }^{*} \mathrm{P}<0.05$. 
Table 3. Patient demographics and clinical characteristics of survivors versus non-survivors in patients presenting with either tachycardia or tachypnea

\begin{tabular}{|c|c|c|c|c|c|c|}
\hline & \multicolumn{3}{|c|}{ Tachycardia } & \multicolumn{3}{|c|}{ Tachypnea } \\
\hline & Died $n=80$ & Survived $n=1,690$ & P-value & Died $n=92$ & Survived $n=1,407$ & P-value \\
\hline Age, yr (SD) & $58(18.6)$ & $52(17.6)$ & $<0.01$ & $65(18.1)$ & 57 (17.9) & $<0.01$ \\
\hline Sex & & & 0.28 & & & 0.19 \\
\hline Male & $45(56.3)$ & 836 (49.5) & & $53(57.6)$ & $704(50.0)$ & \\
\hline Female & 35 (43.8) & $854(50.5)$ & & $39(42.4)$ & $703(50.0)$ & \\
\hline \multicolumn{7}{|l|}{ Race } \\
\hline White & $38(47.5)$ & $786(46.5)$ & 0.92 & $53(58.2)$ & $659(46.8)$ & 0.25 \\
\hline African American & $40(50.0)$ & 875 (51.8) & 0.86 & $37(40.7)$ & $724(51.5)$ & 0.22 \\
\hline Asian & $2(2.5)$ & $13(0.8)$ & 0.10 & $1(1.1)$ & $10(0.7)$ & 0.69 \\
\hline Other & $0(0)$ & $16(0.9)$ & 0.38 & $1(1.1)$ & $14(1.0)$ & 0.44 \\
\hline Ethnicity & & & 0.99 & & & 0.78 \\
\hline Hispanic & $3(3.8)$ & $65(3.8)$ & & $3(3.3)$ & $39(2.8)$ & \\
\hline Non-hispanic & 77 (96.3) & $1,625(96.2)$ & & 89 (96.7) & $1,368(97.2)$ & \\
\hline Hypertension & $24(30.0)$ & 713 (42.2) & 0.03 & $32(34.8)$ & $698(49.6)$ & $<0.01$ \\
\hline Diabetes & $20(25.0)$ & $453(26.8)$ & 0.72 & $23(25.0)$ & 411 (29.2) & 0.39 \\
\hline CHF & $7(8.8)$ & $153(9.1)$ & 0.93 & $20(21.7)$ & $210(14.9)$ & 0.08 \\
\hline End-stage renal disease & $9(11.3)$ & $112(6.6)$ & 0.11 & $7(7.6)$ & $79(5.6)$ & 0.43 \\
\hline Cirrhosis/hepatitis & $3(3.8)$ & $87(5.1)$ & 0.58 & $4(4.3)$ & $66(4.7)$ & 0.88 \\
\hline Stroke & $9(11.3)$ & $106(6.3)$ & 0.08 & $12(13.0)$ & $111(7.9)$ & 0.08 \\
\hline Asthma/COPD & $15(18.8)$ & $301(17.8)$ & 0.83 & $22(23.9)$ & $325(23.1)$ & 0.86 \\
\hline Malignancy (active) & $10(12.5)$ & $94(5.6)$ & & 7 (7.6) & $68(4.8)$ & \\
\hline HIV/AIDS & $5(6.3)$ & $113(6.7)$ & 0.88 & $6(6.5)$ & $74(5.3)$ & 0.60 \\
\hline White blood count, cells per mm3 (SD) & $16(18.8)$ & $11(5.7)$ & $<0.01$ & $15(16.1)$ & $10(5.2)$ & $<0.01$ \\
\hline Hemoglobin, mg/dL (SD) & $12(2.9)$ & $13(2.4)$ & 0.01 & $12(2.9)$ & $13(2.3)$ & 0.02 \\
\hline Bicarbonate, mg/dL (SD) & $22(5.2)$ & $25(4.8)$ & $<0.01$ & $23(5.6)$ & $25(4.8)$ & $<0.01$ \\
\hline Creatinine, mg/dL (IOR) & $1.4(1.0-2.2)$ & $1.1(0.8-1.5)$ & $<0.01$ & $1.5(1.1-2.4)$ & $1.1(0.9-1.6)$ & $<0.01$ \\
\hline Intravenous fluid, mL (SD) & $1,397(1,377.0)$ & $877(1,053.0)$ & $<0.01$ & $1,114(1,368.0)$ & $692(977.0)$ & $<0.01$ \\
\hline Temporarily normalized & $26(32.5)$ & $803(47.5)$ & $<0.01$ & $39(42.4)$ & $821(58.4)$ & $<0.01$ \\
\hline Normal upon admission & $21(26.3)$ & 675 (39.9) & 0.01 & $32(34.8)$ & $699(49.7)$ & 0.01 \\
\hline Any hypotension & $34(42.5)$ & 349 (20.7) & $<0.01$ & $42(45.7)$ & 276 (19.6) & $<0.01$ \\
\hline
\end{tabular}

Values are presented as number (\%) unless otherwise indicated.

SD, standard deviation; CHF, congestive heart failure; COPD, chronic obstructive pulmonary disease; HIV/AIDS, human immunodeficiency virus/acquired immunodeficiency syndrome; IQR, interquartile range.

Table 4. Unadjusted and adjusted odds ratios for in-hospital mortality among patients with and without persistent tachycardia and tachypnea

\begin{tabular}{llcccc}
\hline & & Unadjusted odds ratio & $95 \% \mathrm{Cl}$ & Adjusted odds ratio & $95 \% \mathrm{Cl}$ \\
\hline Persistent tachycardia & All vital sign measurements & 1.88 & $1.17-3.03$ & 1.81 & $1.06-3.09$ \\
& Upon admission & 1.87 & $1.12-3.10$ & 1.77 & $1.00-3.12$ \\
& Hypotension & 2.84 & $1.80-4.50$ & 2.18 & $1.29-3.67$ \\
Persistent tachypnea & All vital sign measurements & 1.90 & $1.24-2.74$ & 1.72 & $1.07-2.74$ \\
& Upon admission & 1.85 & $1.19-2.88$ & 1.67 & $1.03-2.70$ \\
& Hypotension & 3.44 & $2.23-5.30$ & 2.74 & $1.70-4.40$ \\
\hline
\end{tabular}

$\mathrm{Cl}$, confidence interval.

(normal versus abnormal) rather than a continuous variable to account for the impact of hypothermia on data analysis. The unadjusted and adjusted odds ratios (with 95\% Cls) for the various measures of vital sign normalization are provided in Table 4, with estimates for the odds ratios associated with at least one occurrence of hypotension provided for comparison. In a post-hoc analysis, we added GCS to our final models to determine the im- pact of this readily available, bedside measure to influence the prediction of mortality. In our multivariate tachycardia model, GCS was indeed an independent predictor of mortality, though all other variables remained significant predictors. When added to the tachypnea model, GCS significantly predicted mortality, but normalization of tachypnea no longer reached statistical significance as an independent predictor of mortality. 


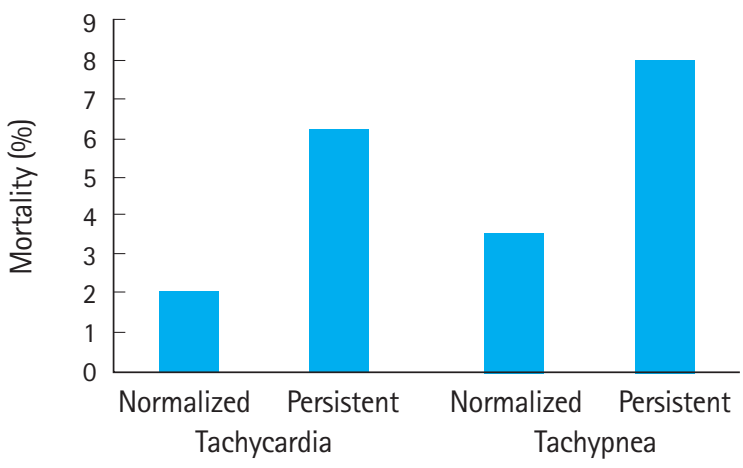

Fig. 2. Relative mortality associated with the persistence of tachycardia and/or tachypnea in patients with both measures abnormal at emergency department triage $(\mathrm{P}=0.08)$. Note that persistent tachypnea tends to carry a worse prognosis than persistent tachycardia.

Finally, we attempted to determine whether persistent tachypnea and tachycardia were independently associated with increased risk of mortality, or whether they were different metrics that identified the same group of patients. Eight hundred and fourteen patients demonstrated both initial tachycardia and tachypnea. Normalization of these two parameters in the ED were independent of each other by chi-square testing $(P<0.001)$. Mortality was highest in patients who had persistence of both tachycardia and tachypnea throughout their ED stay at $8.1 \%$, and was lowest among patients who normalized both (3.9\%) as illustrated in Fig. 2. However, these results did not reach statistical significance in the stratified analysis $(P=0.08)$.

\section{DISCUSSION}

In this cross-sectional single center study of ED patients, we document the increased mortality associated with persistence of tachycardia and tachypnea. Vital sign measurements are the cornerstone of clinical practice, and provide a simple fundamental method to assist in the evaluation of patients. In our study, we found mortality rates for patients with persistent tachycardia and tachypnea at the time of admission were nearly doubled, for an absolute increased risk of mortality of 3\% to $4 \%$, compared to if these vital signs returned to a normal range. Similarly, even transient normalization of these vital signs are associated with an improved prognosis compared to patients who never have a normal heart or respiratory rate measured in the ED. The prognostic value persisted even when several different cutoffs for the definitions of tachycardia and tachypnea were investigated. These findings support the anecdotal practice of using normalization of vital signs for risk assessment of ED patients.

Several findings of our study warrant further consideration. It would be reasonable to assume that patients failing to normalize these vital signs would be likely to have other indications of illness, such as hypotension, and that the relative utility of vital sign normalization might be negligible. Our results were significant exclusive of measurements of hypotension, though the absolute risk of death was lower following exclusion of hypotensive patients. As illustrated by Table 4, any episode of hypotension remains a stronger indicator of mortality (odds ratios of 2.74 and 3.44). However, the risk associated with normalization of heart rate and respiratory rate remains significant even after controlling for the presence of hypotension and other clinical predictors of mortality. Combined, these data suggest that heart rate and respiratory rate normalization may be a particularly useful indicators in the cohort who require hospital admission but who do not exhibit hemodynamic instability through the manifestation of hypotenstion in the ED. Similarly, altered mental status is a readily clinically available indicator with established utility to predict patient outcome in ED patients. ${ }^{10,11}$ When included in our multivariate model, persistent tachycardia remained a significant independent predictor of mortality, supporting its clinical utility. Persistent tachypnea was no longer statistically significant after addition of GCS our model, however. It could be that patients with significantly altered mental status were more likely to be intubated with resultant artificial respiratory rates. This confounder may have affected our study results, though that data was not recorded, making interpretation of persistent tachypnea less clear than tachycardia.

It is important to note that the absolute mortality rates in the study are low, but are consistent with the mortality rate of an unselected cohort of patients admitted to a tertiary care facilities. We found the absolute mortality rate of either persistent tachycardia or tachypnea to be similar to that of a single measured episode of hypotension, approximately 5\% in this cohort. However, this mortality risk is lower than those patients that exhibit episodic or persistent hypotension ( $8 \%$ and $14 \%$, respectively). ${ }^{6}$ The present findings are not surprising given the fact that changes in heart and respiratory rate often precede hypotension, though perhaps these changes might serve as an early warning sign of clinical decompensation for clinicians.

A critical finding of the study worth noting for clinicians is the observation that, as a univariate predictor, the absolute risk of mortality associated with persistent tachypnea is significantly higher than that associated with persistent tachycardia. Given the role of the respiratory system for the buffering of acidosis, this finding is biologically plausible. Previous studies have suggested that respiratory rates recorded in the medical record are frequently inaccurate, ${ }^{12}$ and our data support the importance of 
accurately assessing and noting respiratory rate for both nursing staff and physicians.

As illustrated in Fig. 2, it remains unclear based on this data whether persistent tachycardia and tachypnea are in fact independent risk factors for mortality or different manifestations of the same underlying physiologic processes. This may be due to the relatively smaller sample size of patients presenting with both tachycardia and tachypnea. The question of whether persistent tachycardia and persistent tachypnea are independent prognostic predictors remains an area for future investigation that is not conclusively resolved in either direction by this study.

There are several limitations that deserve consideration. First, patients had an average of $>4$ sets of vital signs measured, and the utility of vital sign normalization among patients with fewer measurements (due to very rapid ED throughput or areas with limited monitoring capacity) or more intensive/continuous monitoring is unknown, which is why we chose to conduct several different sensitivity analyses to assess the robustness of our findings. Similarly, tachypnea was recorded by bedside nurses using conventional monitors, and it is possible this method introduced increased variability compared to more standardized measurements taken by counting breaths or influenced by an anecdotal tendency to record "normal" values. However, either of these scenarios would bias our results towards the null, and therefore are unlikely to invalidate the results of our study. Second, this is a single center study and the data were gathered approximately 10 years ago, and results may not be generalizable to other settings. Third, it is possible that this same information could be gathered through the use of other prognostic clinical tests, such as lactate ${ }^{13}$ or base excess. ${ }^{14}$ However, we did not systematically evaluate these measurements in our study. Similarly, while we hypothesize that persistently abnormal vital signs may be early markers of impending hemodynamic instability, these data were not tracked and that hypothesis not specifically tested in this study. We did not record data regarding ED disposition, elevation in level of care, or short-term deterioration outcomes such as cardiac arrest or endotracheal intubation, and follow-up studies to determine the ability of vital sign normalization to predict these outcomes may be warranted. Critically, the design of our study only allows us to reach conclusions regarding prognosis. While it would be tempting to extrapolate these findings to suggest that the use of vital sign trends might be used to help determine the level of inpatient monitoring and care, such a conclusion would be premature and requires future investigation. Finally, all patients in this study were admitted to the hospital, and whether normalization of vital signs carry similar prognostic potential in patients discharged from the ED remains unclear.
Persistent tachycardia or tachypnea are associated with an increased risk of mortality in ED patients admitted to the hospital, particularly in those without hypotension. Further study is necessary to determine if interventions aimed at improved recognition or earlier intervention in this group of patients can decrease mortality.

\section{CONFLICT OF INTEREST}

No potential conflict of interest relevant to this article was reported.

\section{ACKNOWLEDGMENTS}

Michael A Puskarich has received salary support from the National Institutes of Health/General Medical Sciences (K23 GM113041-01).

\section{REFERENCES}

1. Centers for Disease Control and Prevention. Emergency department visits [Internet]. Atlanta, GA: Centers for Disease Control and Prevention; 2015 [cited 2015 Jan 14]. Available form: http://www.cdc.gov/nchs/fastats/emergency-department.htm.

2. Hoot NR, Aronsky D. Systematic review of emergency department crowding: causes, effects, and solutions. Ann Emerg Med 2008;52:126-36.

3. McConnell KJ, Richards CF, Daya M, Bernell SL, Weathers CC Lowe RA. Effect of increased ICU capacity on emergency department length of stay and ambulance diversion. Ann Emerg Med 2005;45:471-8.

4. Jones $A E$, Aborn $L S$, Kline JA. Severity of emergency department hypotension predicts adverse hospital outcome. Shock 2004:22:410-4.

5. Jones $A E$, Stiell IG, Nesbitt LP, et al. Nontraumatic out-ofhospital hypotension predicts inhospital mortality. Ann Emerg Med 2004;43:106-13.

6. Jones AE, Yiannibas V, Johnson C, Kline JA. Emergency department hypotension predicts sudden unexpected in-hospital mortality: a prospective cohort study. Chest 2006;130:941-6.

7. Kaukonen KM, Bailey M, Pilcher D, Cooper DJ, Bellomo R. Systemic inflammatory response syndrome criteria in defining severe sepsis. N Engl J Med 2015;372:1629-38.

8. Rangel-Frausto MS, Pittet $D$, Costigan $M$, Hwang T, Davis CS, Wenzel RP. The natural history of the systemic inflammatory response syndrome (SIRS): a prospective study. JAMA 1995; 273:117-23.

9. Brun-Buisson C. The epidemiology of the systemic inflamma- 
tory response. Intensive Care Med 2000;26 Suppl 1:S64-74.

10. Vincent JL, Moreno R, Takala J, et al. The SOFA (Sepsis-related Organ Failure Assessment) score to describe organ dysfunction/failure. On behalf of the Working Group on Sepsis-Related Problems of the European Society of Intensive Care Medicine. Intensive Care Med 1996;22:707-10.

11. Shapiro NI, Wolfe RE, Moore RB, Smith E, Burdick E, Bates DW. Mortality in Emergency Department Sepsis (MEDS) score: a prospectively derived and validated clinical prediction rule. Crit Care Med 2003;31:670-5.
12. Lovett PB, Buchwald JM, Sturmann K, Bijur P. The vexatious vital: neither clinical measurements by nurses nor an electronic monitor provides accurate measurements of respiratory rate in triage. Ann Emerg Med 2005;45:68-76.

13. Jansen TC, van Bommel J, Bakker J. Blood lactate monitoring in critically ill patients: a systematic health technology assessment. Crit Care Med 2009;37:2827-39.

14. Middleton P, Kelly AM, Brown J, Robertson M. Agreement between arterial and central venous values for $\mathrm{pH}$, bicarbonate, base excess, and lactate. Emerg Med J 2006;23:622-4. 